\title{
Mosaic structure of the emission region of pulsar PSR $0329+54$
}

\author{
A.D.Kuzmin, V.A.Izvekova \\ Astro Space Center of Lebedev Physical Institute, Russia
}

\begin{abstract}
We performed the multifrequency time aligned measurements and the component structure analysis of the integrated profile of PSR $0329+54$ in frequency range from 0.1 to $10 \mathrm{GHz}$. The result is that a commonly adopted five-component structure of the integrated profile does not provide a good match to the observed profiles. Only a six-component structure fits observations well. This result calls into question the validity of the two conal and core zones emission model. We suggest that the emission region inside of the cone of the open magnetic field lines represents a mosaic bunch of the discrete outflows of relativistic charges along magnetic field lines, injected by mosaic group of localized sparks in the polar cap. Our suggestion is based on the Rutherman and Sutherland belief that the polar gap discharges through a group of localized sparks, spaced at distances nearly equal to the height of the polar gap.

Mode changing may be produced by the change of an activity of some sparks pattern without rearranging the structure of the emission region.
\end{abstract}

\section{Introduction}

The general source of the structural information of the pulsar emission region is the multifrequency observations of the integrated pulse profiles. In the present work we analyze the component structure of the integrated profile and the emission region of the pulsar PSR $0329+54$.

\section{Observations and component structure analysis}

We used multifrequency time alignment collaborative observations, performed in Pushchino Observatory, Russia at $102 \mathrm{MHz}$, Jodrell Bank, UK at 230,408, 610 and $1380 \mathrm{MHz}$ and Effelsberg, Germany at 1.4,4.7 and $10.5 \mathrm{GHz}$.

To perform a component structure analysis in an objective and quantitative way we used the Gaussian fit separation of the average profile method, outlined by $\mathrm{Wu}, \mathrm{Xu}$ and Rankin (1992); Foster, Fairhead and Backer (1991). The integrated profile was presented as a superposition of Gaussian-shaped individual components

$$
I(\phi)=\sum_{i=1}^{n} a_{k} \exp \left[-\ln 2\left(\left(\phi-\phi_{k}\right) / w_{k}\right)^{2}\right]
$$


where $a_{k}, w_{k}$ and $\phi_{k}$ are the intensity, half-power width and phase position of the components. The analytical profile $I(\phi)$ was compared with the observed one to obtain the minimal residual between these profiles by a least square iterative fitting procedure and to find the $a_{k}, w_{k}$ and $\phi_{k}$ values that match the observed and analytical profiles best. The number of components was adopted as initial parameters. All the other parameters were derived in the fitting procedure.

The most critical point of the fitting analysis is the proper choice of the number of Gaussian components. We apply as the choosing criterion that in a good fit the residual (the difference between the observed and the analytical profiles) should be near the off-pulse noise. This means that the residual should not have any regular component-like structures, which exceed the off-pulse noise, and the value of the residual noise should be about the same as the value of the off-pulse noise. If the residual has a regular structure and the residual noise exceeds noticeably the off-pulse noise an additional component(s) is required.

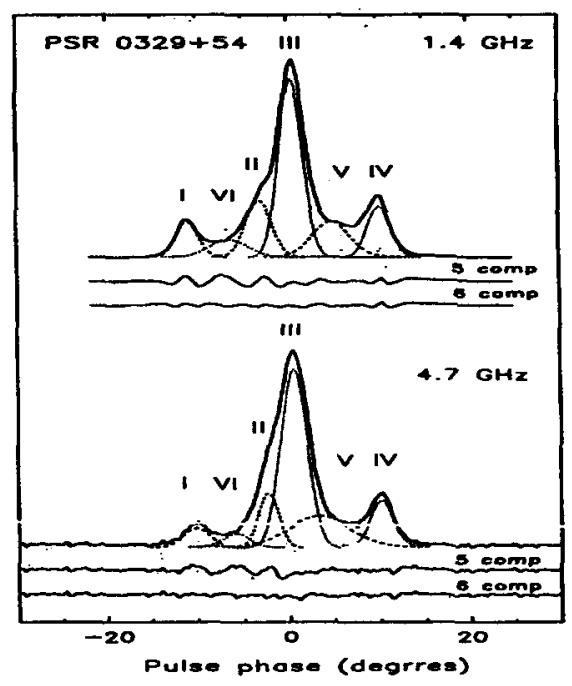

Figure 1. Gaussian fit to the integrated profile at 1.4 and $4.7 \mathrm{GHz}$ assuming five and six component structure. Solid line- the observed profiles, dotted lines- the Gaussian components. Beneath the profiles there are residual curves for five and six components.

As the first approach we adopt five components structure as commonly adopted for this pulsar (Hesse 1973). But this choice did not provide a good fit over all the observations frequencies. The residual has considerable componentlike structure details between components I and II and the residual noise is much larger than the off-pulse noise. This is illustrated in Fig. 1 for profiles at $1.4 \mathrm{GHz}$ and $4.7 \mathrm{GHz}$. The solid lines are the observed profiles and doted and dashed lines are the components. Under each profile we plot the residual curves for five and six components. For five components one can see the residual component- 
like structure details between components I and II. The excess of the residual noise over the off-pulse noise at these frequencies is about 3 times.

For good fitting one needs to add the additional component between components I and II. Six component structure diminished the residual to the off-pulse noise level and provides a good fit to observed profiles. The similar, but less pronounced picture is at other frequencies. Only a six-component structure fits observations well.

We observed several cases of the mode changing at $102 \mathrm{MHz}, 1.4$ and 10.7 $\mathrm{GHz}$. Mode changing appears as the burst of some components. In all cases the number and position of the components do not vary, only the relative intensities do.

\section{Discussion}

A commonly adopted five-component structure of the integrated profile of PSR $0329+54$ does not provide a good match to the observed profiles. Only a sixcomponent structure fits the observations well. This result call into question the validity of the two conal and core zones emission model (Oster and Sieber, 1977; Rankin 1983). Phenomenologicaly one may suggest three cone emission zones model. We prefer an another approach.

We suggest that the emission region inside of the cone of the open magnetic field lines represent a mosaic bunch of the discrete outflows of relativistic charges along magnetic field lines, injected by mosaic group of localized sparks in the polar cap (Fig.2).

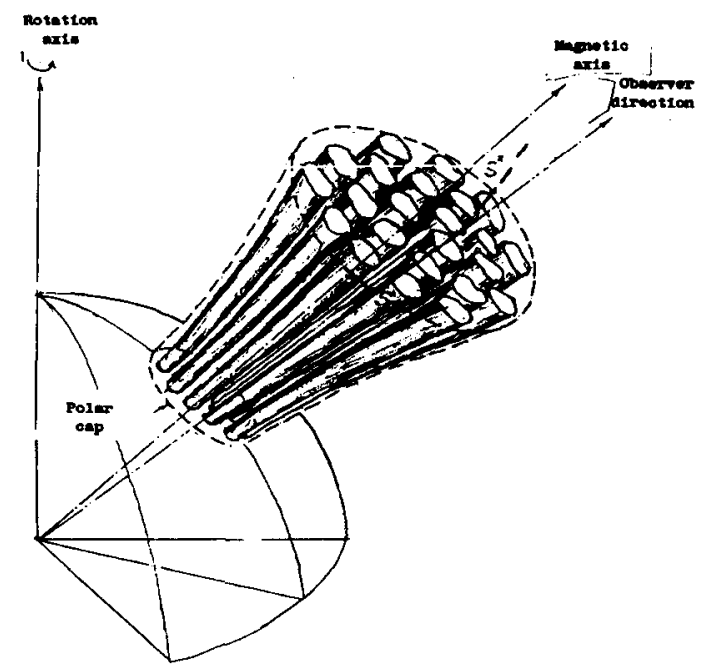

Figure 2. Structure of the emission region. The solid dashed line SS' is the section of the emission region seen by an observer. 